\title{
Joint Optimization of Interference and Cost in Cognitive Radio Heterogeneous Network Using Fuzzy Logic Powered Ants
}

\author{
Shahzad Latif, ${ }^{1,2}$ Suhail Akraam, ${ }^{3}$ and Muhammad Aamer Saleem ${ }^{4}$ \\ ${ }^{1}$ School of Engineering \& Applied Sciences, Isra University, Islamabad, Pakistan \\ ${ }^{2}$ Shaheed Zulfikar Ali Bhutto Institute of Science and Technology (SZABIST), Islamabad, Pakistan \\ ${ }^{3}$ International Islamic University, Islamabad, Pakistan \\ ${ }^{4}$ Hamdard Institute of Engineering \& Technology, Hamdard University, Islamabad, Pakistan \\ Correspondence should be addressed to Shahzad Latif; shahzadlatif97@gmail.com
}

Received 24 April 2017; Revised 2 August 2017; Accepted 16 August 2017; Published 9 October 2017

Academic Editor: Xianfu Lei

Copyright (c) 2017 Shahzad Latif et al. This is an open access article distributed under the Creative Commons Attribution License, which permits unrestricted use, distribution, and reproduction in any medium, provided the original work is properly cited.

\begin{abstract}
The advent of emerging wireless technologies has increased the demand of spectrum resources. On the other hand, present spectrum assignment is fixed and underutilized. Cognitive radio (CR) provides good solution to spectrum scarcity problem to accommodate new wireless applications. The network selection is an important mechanism in cognitive radio heterogeneous network (CRHN) to provide optimal Quality of Service (QoS) to both Primary Users (PUs) and Secondary Users (SUs). The aim of this work is to provide optimal QoS to SUs by appropriate network selection and channel assignment. The proposed FLACSA selects the best network while maximizing the data rate and minimizing the interference and cost simultaneously. Simulation results show the attractive performance of our proposed algorithm.
\end{abstract}

\section{Introduction}

Data transmission over wireless channel has been increased exponentially during the last decade. Due to this heavy load, wireless systems are facing problem of spectrum scarcity. To solve this problem, either expand wireless spectrum or use available spectrum efficiently and intelligently. Fifthgeneration $(5 \mathrm{G})$ networks are expected to provide high data rates with good QoS. Thus, in the coming years, demand for high data rates will increase manyfold. There are different views about $5 \mathrm{G}$ architecture: how to cope with high data rates such as cognitive radios, small cells, light communication, and MIMO communication systems. The 5G networks are considered as a heterogeneous network that consists of different types of primary networks. The small cells deployment can meet the demand of high data rates in 5G networks. Moreover, efficient spectrum sharing temporally and spatially ensures the coverage of $5 \mathrm{G}$ networks everywhere and all the time. CRN opportunistically utilized the spectrum of licensed networks (primary network). CRNs are becoming the best choice to use wireless spectrum efficiently [1]. CRNs use the spectrum holes available in existing wireless networks. The unlicensed users of CRN are known as SUs and licensed users of primary networks are known as PUs [2].

This may face a problem in selecting which licensed network to join because CRHN incorporates multiple primary networks with different price and capacity requirements. In CRHN, selection of a suitable network [3], keeping in view the price, interference, and capacity, is thus very important [4, 5]. Authors in [6] considered a network selection problem as a noncooperative game in which SUs were considered as players that achieved equilibrium without cooperation. Markov decision process is proposed in [7] to maximize SUs throughput in a cognitive radio heterogeneous network. Many techniques, such as game theory based spectrum allocation [8], pricing, and auction mechanism based spectrum assignment [9-11], have been developed in the literature on spectrum assignment in CRHN. Multiobjective optimization is NPhard problem in cognitive radio networks [12].

Evolutionary computing algorithms are good to approximate NP-hard problems due to their low computational complexity [12]. Evolutionary algorithms are inspired from 
biological behaviors of different living organisms. In [13], particle swarm optimization (PSO) is proposed for sensing in CRNs. Genetic Algorithm (GA) and PSO are used for resource allocation. A differential evolution based channel assignment algorithm is designed for CRNs in [14]. In [15], GA and PSO are investigated to optimize price and interference in CRHN. The authors in [15] suggested that the GA can find optimal solution in comparison with PSO algorithm under the constraints on interference, data rate, and price. However, SUs accumulative data rates optimization and impact of varying densities of SUs on QoS are not well explored.

In this research work, we consider a heterogeneous network that consists of different types of primary networks. Each primary network has its own constraints regarding interference, data rates, and cost to buy the spectrum.

The main contributions of the paper are listed as follows:

(i) We formulate an optimization problem in which the objective is to maximize data rates of SUs and minimize the interference incurred to PUs. Moreover, overall cost to buy primary network resources is also minimized.

(ii) A fuzzy logic ant colony system algorithm (FLACSA) has been proposed to optimize network selection and channel assignment problem in order to provide QoS to SUs.

(iii) We compare our method with other studied algorithms in literature. Numerical results show that the proposed method achieves superior performance in comparison with the other algorithms.

(iv) The impact of varying densities of SUs on cost, interference, and data rates of SUs in CRHN is well investigated.

The rest of the paper is organized as follows. The heterogeneous network model is explained in Section 2. The FLACSA based optimization problem is formulated in Section 3. Section 4 presents the simulation results and discussion. Finally, the research work is concluded in Section 5.

\section{Heterogeneous Network Model}

The CRHN consists of $N$ primary networks. Each primary network consists of different number of PUs as shown in Figure 1. Availability of channels to SUs depends on the behavior of PUs. All primary networks are connected to a centralized operating mechanism that knows requirements of SUs and PUs activity. It is assumed that communication environment is slowly varying during spectrum decision time.

The centralized monitoring mechanism gathered the information such as number of SUs $M$ and number of free available channels $W$ in primary networks. It is assumed that $j$ th SU incurred unit interference $h_{j i}$ to $i$ th PU.
The objectives are

(i) minimizing the cost paid by SU,

(ii) minimizing the interference provided by SU,

(iii) maximizing the accumulative data rates by SUs.

In mathematical form,

$$
\begin{array}{ll}
\operatorname{minimize} & R(x)=\sum_{j=1}^{M} \sum_{i=1}^{N}\left(h_{j i}+f_{j i}\right) x_{j i} \\
\operatorname{maximize} & S(x)=\sum_{j=1}^{M} \sum_{i=1}^{N} \gamma_{j i} x_{j i},
\end{array}
$$

where $R(x)$ denotes accumulative function of interference and cost, $S(x)$ is accumulative function of SUs overall data rates, $f_{j i}$ is cost to buy the $i$ th network by the $j$ th SU, and $\gamma_{j i}$ represents $j$ th SU data rate requirement in $i$ th network.

Subject to constraints, we have the following:

(i) Each SU is given only one channel at a time.

$$
\sum_{i=1}^{N} x_{j i}=1, \quad \forall j=1,2, \ldots, M,
$$

where $x$ represents binary decision variable. If $x_{j i}=1$, the $j$ th SU gets the channel of $i$ th network and vice versa.

(ii) The total interference caused by the SUs to $i$ th network should be below the threshold level $\epsilon_{i}$.

$$
\sum_{j=1}^{M} h_{j i} x_{j i} \leq \epsilon_{i}, \quad \forall i=1,2, \ldots, N, j=1,2, \ldots, M
$$

(iii) The data rate $\gamma_{j}$ of $j$ th SU should be less than or equal to data rate $C_{k i}$ of $i$ th network using $m$ th channel.

$$
\gamma_{j} x_{j i} \leq C_{k i} \quad \forall i=1,2, \ldots, N, k=1,2, \ldots, W .
$$

(iv) The price $p_{j}$ paid by $j$ th SU should be equal to or greater than the price $f_{i}$ to buy the $i$ th network.

$$
p_{j} x_{j i} \geq f_{i}, \quad \forall i=1,2, \ldots, N, j=1,2, \ldots, M .
$$

The accumulative functions $R(x)$ and $S(x)$ as described in (1a) and (1b) are considered as fitness function and are used to evaluate the performance of the proposed algorithm.

\section{Proposed Fuzzy Logic Ant Colony System Algorithm (FLACSA)}

The ants have the ability to search the shortest path between nest and food source by releasing a liquid substance called pheromone on their routes. This behavior was first investigated by Dorigo et al. [16].

The fuzzy logic (FL) was introduced by Zadeh [17]. Fuzzy systems have been making rapid progress in the recent 


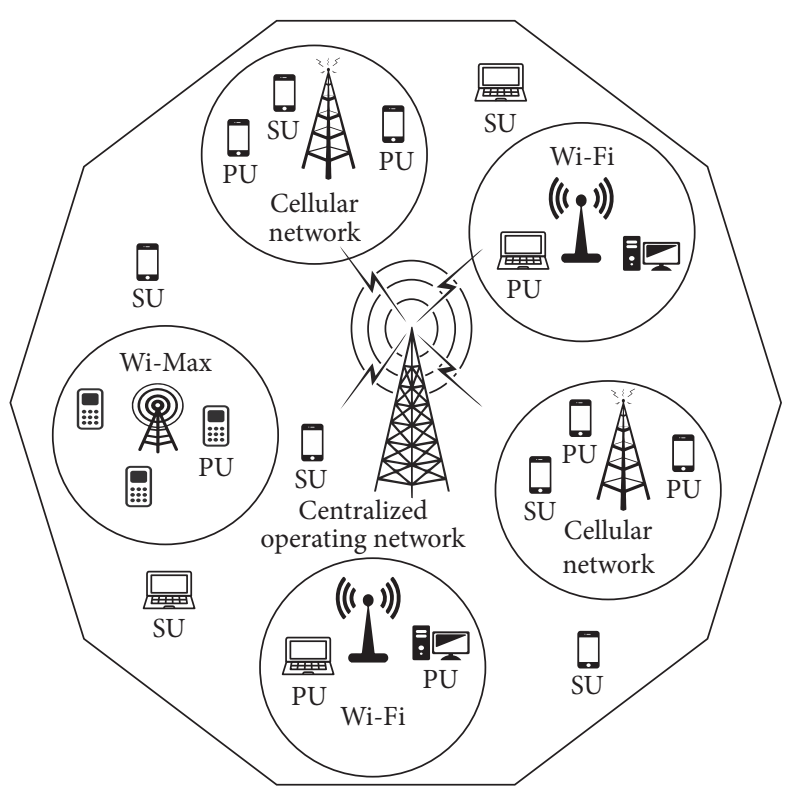

FIGURE 1: Cognitive radio heterogeneous network model.

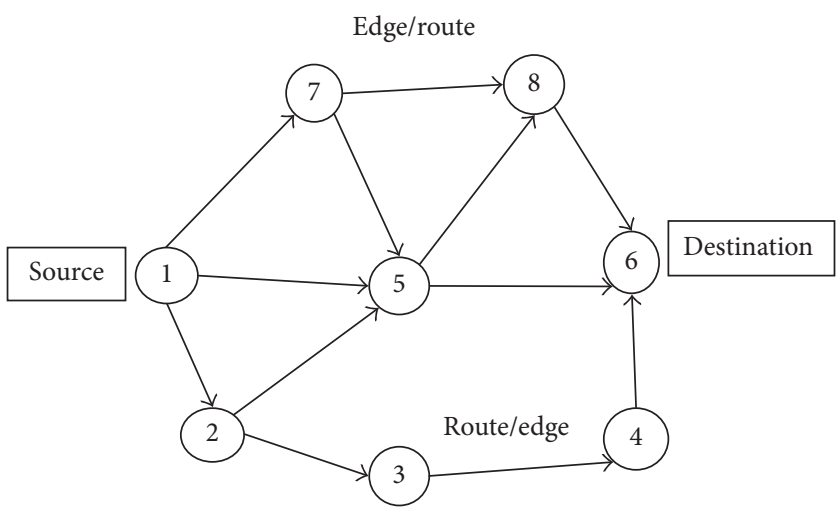

Figure 2: Ant colony graph.

years. According to fuzzy logic (FL), an object can take any real value between 0 and 1. Fuzzy control can be defined by linguistic sentences called IF-THEN rules instead of mathematical equations. The process of deduction of rules is called inference [18]. Fuzzy logic controlling system stages are as follows: (a) Fuzzification operation can map mathematical input values into fuzzy membership functions. (b) In next step, linguistic rules are made to execute fuzzy membership functions. (c) Defuzzification operation is used to map a fuzzy output into binary form called crisp output values. There are different defuzzification techniques; for example, the center of gravity and the mean of maxima are mostly used $[18,19]$.

In the proposed FLACSA, SUs are represented by ants and licensed networks are denoted by nodes as shown in Figure 2. A colony of ants is considered on each node. In this figure, the interference to networks by SUs is represented by edges. Based on the pseudocode in Algorithm 1, the search process runs for each node, respectively. In each journey, a traveler ant collects information of the transited edges and visited nodes. After arriving at a destination, based on the analyses of transited path by the fuzzy system, the ant releases pheromone on each edge. This procedure will be repeated for all ants, noting that the result of the mentioned procedure is a pheromone report, which presents pheromone amount of each node for each route. Different steps of the proposed algorithm are described as in Algorithm 1.

3.1. Initialization. Initial parameter values are set at this stage. SU $m^{\prime}$ aims at network $n^{\prime}$. Topology information as number of SUs $M$ and primary networks $N$, each with $w_{m}$ channels, is imported to the algorithm regularly using centralized 


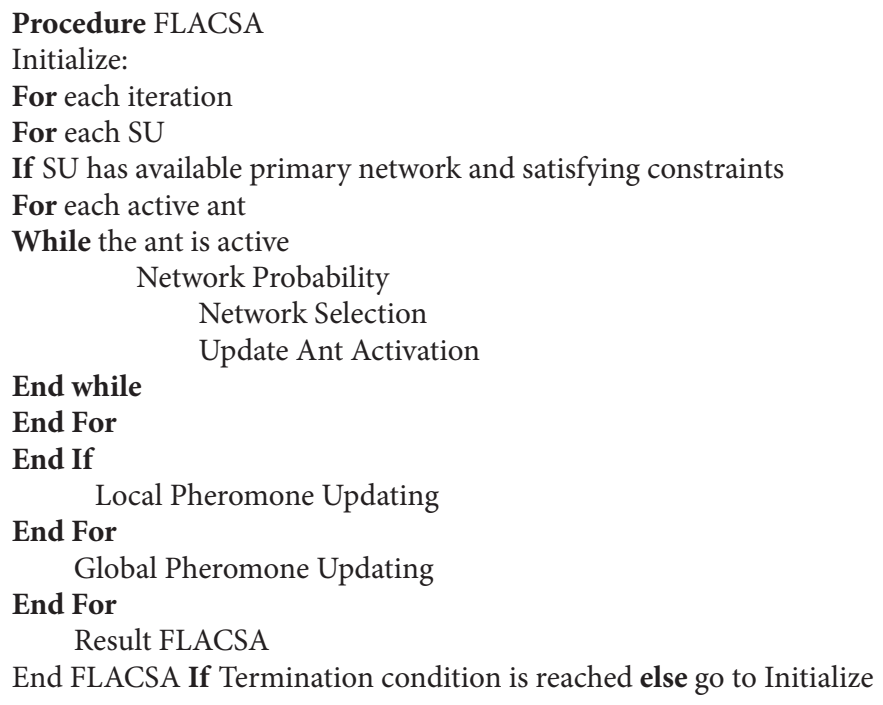

Algorithm 1: General description of FLACSA.

operative network. It is assumed that each network has the same number of channels. The active ants are also located at each node, respectively, to start their journey at this step.

3.2. Network Probability. After being located at a node in each iteration, each ant must select an edge to continue its journey. The selection probability $P$ of each candidate edge $i$ for active ant $A$ is expressed as follows:

$$
P_{i}^{A}=\left\{\begin{array}{cc}
0 & \forall \sum_{j=1}^{N} L_{m^{\prime}, j}^{\prime}=0 \\
\frac{T_{n^{\prime}, i}^{\alpha} \Delta_{i}^{\beta}}{\sum_{i=1}^{I}\left[T_{m, i}^{\alpha} \Delta_{i}^{-\beta}\right]}, & \text { otherwise }
\end{array}\right\} \quad \forall i \in \Gamma_{1 \times I}^{\prime},
$$

where $I$ is the number of potential edges, $\Gamma_{1 \times I}^{\prime}$ represents a list of possible edges, and $L_{m^{\prime}, j}^{\prime}$ is a binary list of available channels for candidate SU $m^{\prime}$. The parameters $\alpha$ and $\beta$ control relative importance of $T_{n^{\prime}, i}$ and $\Delta_{i}$, respectively. $T_{n^{\prime}, i}$ is the pheromone amount of SU $m^{\prime}$ using channel $i$ and $\Delta_{i}$ is the cost of candidate edge for active ant $A$.

3.3. Network Selection. After calculation of each possible edge, an edge must be selected. To do this, random parameter $r_{0}$ with uniform distribution in $[0,1]$ is compared with the predefined parameter $r$, which is set to 0.9 based on trial and error [20]. The results pick up one of the following selection methods:

$$
n^{\prime}=\left\{\begin{array}{cc}
\arg \max \left(P_{i}^{A}\right), & r>r_{0} \\
\text { Roulette Wheel }\left(P_{i}^{A}\right), & \text { otherwise }
\end{array}\right\},
$$

where $n^{\prime}$ is the selected edge. Therefore, the active ant continues its journey through the selected edge to the next node.

3.4. Update Ant Activation. In this algorithm, each ant can visit each node just once. If the current active ant $A$, after its move towards $m^{\prime}$, has no possible edge to continue its journey or $\mathrm{SU} m^{\prime}$ has no interference, this ant is blocked. The activation list

$$
\Omega_{A, m^{\prime}, \xi}=\left\{\begin{array}{ll}
0, & \sum_{j=1}^{M} h_{j m} x_{j m} \leq \epsilon_{m} \\
1, & \text { otherwise }
\end{array}\right\},
$$

where $\Omega_{A, m^{\prime}, \xi}=0$ indicates that ant $A$ is blocked and is considered as a passive ant in the current iteration $\xi$. In this case, the termination condition is met for this ant.

3.5. Local Pheromone Updating. At this stage, some pheromone must be released on the transited edges by active ant $A$. The pheromone amount $T$ of the selected network $n^{\prime}$ for the SU $m^{\prime}$ is updated by

$$
T_{m^{\prime}, n^{\prime}, \xi}^{\mathrm{new}}=T_{m^{\prime}, n^{\prime}, \xi}^{\mathrm{old}}+(\phi \times \Delta T),
$$

where $\Delta T$ is the amount of local pheromone updating managed by an FL system and its impact can be adjusted by $\phi$. Figure 3 illustrates the structure of the FL system, considering Mamdani's implication approach. This model manages $\Delta T$ as output of the FL system by considering two inputs: cost to pay network and interference to the network by SUs. Total cost of the edges visited by each ant is

$$
\Delta_{\text {Total }}^{A}=\sum_{\forall j \in \mathrm{LV}} \Delta_{j}
$$




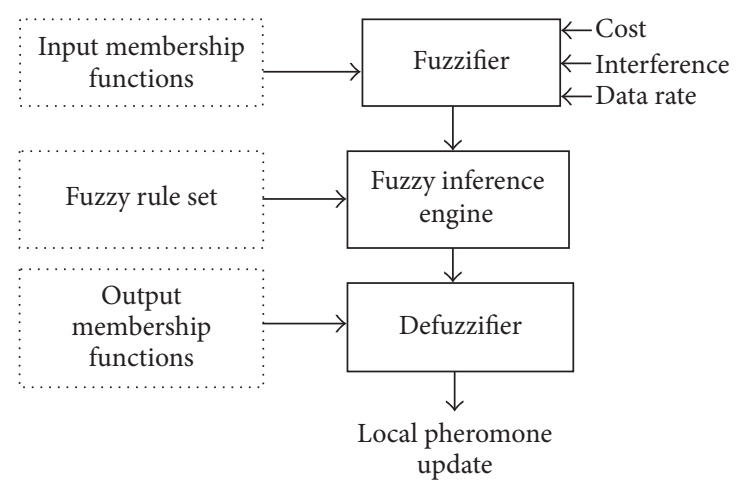

FIgURE 3: Structure of fuzzy logic system for the FLACSA.

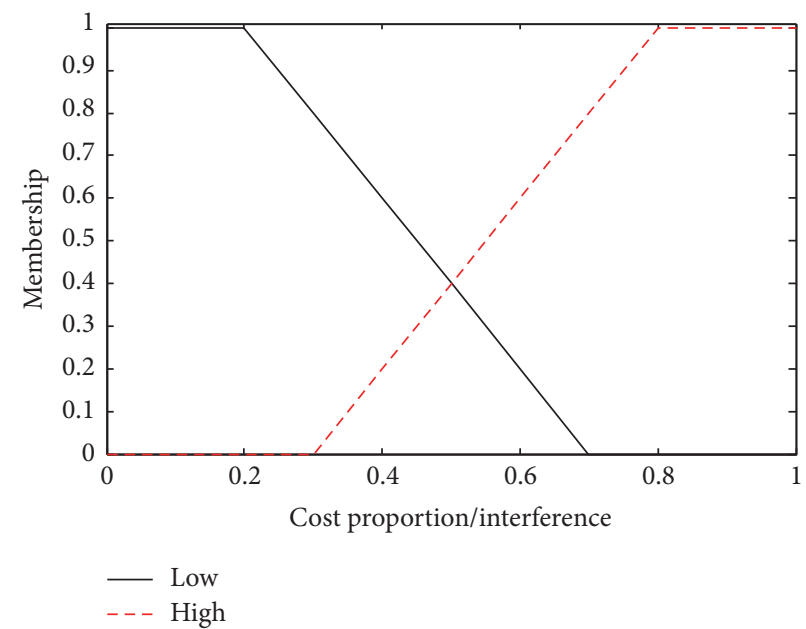

Figure 4: Membership functions of cost, interference, and data rates.

where LV is the list of the visited edges by ant $A$ through its journey. The cost proportion of each ant is obtained by

$$
\Delta_{\mathrm{CP}}^{A}=\frac{\Delta_{\mathrm{Total}}^{A}}{\max \Delta_{\text {Total }}^{A^{\prime}}} \text { for } A^{\prime}=1,2, \ldots, X,
$$

where routes with high $\Delta_{\mathrm{CP}}^{A}$ are not desired by the system and are punished by gaining few $\Delta T ; X$ represents the total number of ants. The total interference is the sum of the interferences on the selected networks through the visited edges and is defined as

$$
h_{\text {Total }}^{A}=\sum h_{j} \quad \forall j \in \mathrm{LV},
$$

where routes with high interference $h_{\text {Total }}^{A}$ are not desired by system and are punished by achieving less $\Delta T$. Fuzzy input membership function is defined by two values, low and high, as shown in Figure 4. Fuzzy output membership function consists of four values, very high, high, low, and very low, respectively, which represent different levels of pheromone as shown in Figure 5.

Based on the above description, Fuzzy Logic IF-THEN rules are illustrated in Table 1 . By considering the rules set, routes with least cost proportion, interference, and high data rates have gained the most local pheromone update. At the final stage, the center of gravity method is employed for the defuzzification process to convert fuzzy values into binary values.

3.6. Global Pheromone Updating. The last traditional step in each completed iteration $\xi$ is global pheromone updating, which is given as follows:

$$
T_{m, n, \xi}^{\text {new }}=\rho T_{m, n, \xi}^{\text {old }} \quad \forall m=1,2, \ldots, M, n=1,2, \ldots, N
$$

where the parameter $0<\rho<1$ is the evaporation coefficient that is set to 0.8 based on trial and error [15].

3.7. Channel Selection. After $\Xi$ number of iterations, based on the pheromone amount, channel $\omega_{m}$ is assigned to $m$ th SU as follows:

$$
\omega_{m}=\arg \max \left(\frac{1}{\Xi} \sum_{\xi=1}^{\Xi} T_{m \times n \times \xi}\right) .
$$




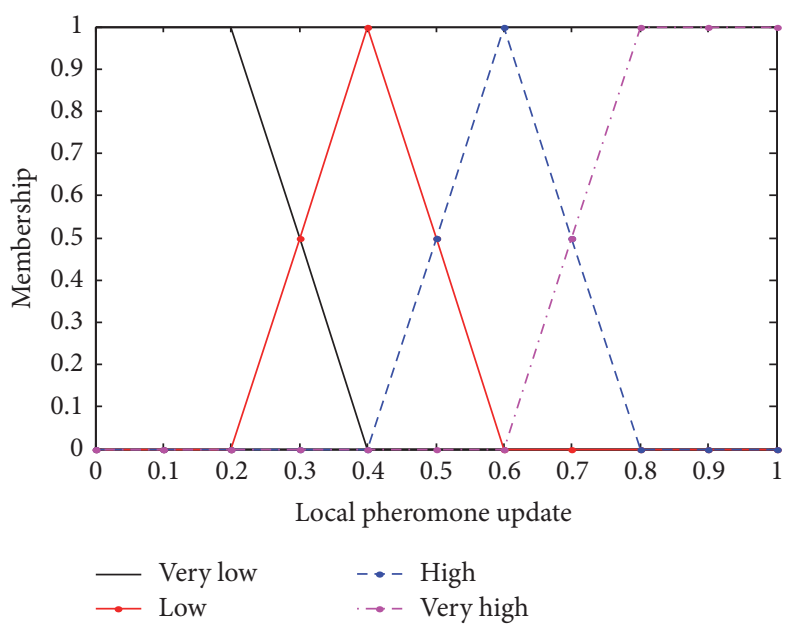

FIgURE 5: Membership function of local pheromone updating.

TABLE 1: Fuzzy IF-THEN rule st for proposed FLACSA.

\begin{tabular}{llccc}
\hline Rule number & Cost & $\begin{array}{c}\text { IF } \\
\text { Interference }\end{array}$ & $\begin{array}{c}\text { THEN } \\
\text { Pheromone update }\end{array}$ \\
\hline$(1)$ & Low & Low & Low & High \\
$(2)$ & Low & Low & High & Very high \\
$(3)$ & Low & High & Low & Low \\
$(4)$ & Low & High & High & High \\
$(5)$ & High & Low & Low & Low \\
$(6)$ & High & Low & High & High \\
$(7)$ & High & High & Low & Low \\
$(8)$ & High & High & High & Very low \\
\hline
\end{tabular}

In this manner, the channel with most obvious pheromone is selected for each SU.

\section{Results and Discussion}

This research work has been conducted using MATLAB software. The performances of FLACSA and ACSA have been evaluated in comparison with PSO and GA approaches. For the objective function with constraints specified in [15], two scenarios, 1 and 2, are considered. In scenario 1 , the price that SUs can pay to buy a network is fixed. In scenario 2, the price to pay by SUs to buy a network is varying. The parameter values for both scenarios, for primary networks and SUs, are presented in Tables 2 and 3, respectively.

By performing initial experiments, parameters are adjusted to achieve high performance. The parameter values for FLACSA are represented in Table 4 for the performed simulations.

Figure 6 shows the impact interference caused by SUs towards PUs in both scenarios 1 and 2, respectively. The results show that FLACSA performs better than all other algorithms under study due to the contribution of FL to updating local pheromone. The average accumulative interference drops to 3 when FLACSA is used; ACSA produces second best results and reduced overall interference of CRHN to 7 in scenario 1 and almost 6 in scenario 2 due to its features of exploration and exploitation in comparison with GA and PSO approaches used in [15].

Figure 7 shows the overall objective function mentioned against number of iterations in scenarios 1 and 2, respectively. Average is taken after 20 experiments. The results show that GA performs well for initial iterations. However, after completing 3000 iterations, FLACSA performs better than all other algorithms and minimized the accumulative cost and interference objective function to 533 in scenario 1 and 562 in scenario 2 .

Figure 8 shows the impact of the cost paid by SUs to buy spectrum resources of primary networks in a CRHN in comparison with number of iterations in scenarios 1 and 2, respectively. FLACSA performs better than GA and PSO after 1100 iterations in scenario 1 and after 800 iterations in scenario 2. During simulation results, it has been observed that FLACSA ensures better results than the GA in large iterations. However, convergence speed of GA is better in starting iterations. 
TABLE 2: Primary networks for scenarios in CRHN.

\begin{tabular}{|c|c|c|c|c|c|c|}
\hline \multirow[t]{2}{*}{ Network ID } & \multicolumn{2}{|c|}{$\begin{array}{l}\text { Cost to join } \\
\qquad\left(f_{m}\right)\end{array}$} & \multicolumn{2}{|c|}{$\begin{array}{c}\text { Capacity per channel } \\
(C)\end{array}$} & \multicolumn{2}{|c|}{$\begin{array}{c}\text { Target interference } \\
(\epsilon)\end{array}$} \\
\hline & 1 & 2 & 1 & 2 & 1 & 2 \\
\hline (1) & 90 & 80 & 80 & 80 & 5 & 7 \\
\hline (2) & 50 & 60 & 100 & 70 & 10 & 6 \\
\hline (3) & 70 & 65 & 70 & 70 & 7 & 7 \\
\hline (4) & 95 & 60 & 100 & 90 & 8 & 10 \\
\hline (5) & 95 & 70 & 80 & 100 & 9 & 9 \\
\hline (6) & 40 & 40 & 80 & 70 & 7 & 5 \\
\hline (7) & 60 & 50 & 80 & 60 & 9 & 9 \\
\hline
\end{tabular}

TABLE 3: SUs requirements and preferences for scenarios.

\begin{tabular}{lcccc}
\hline SU & \multicolumn{2}{c}{ Scenario 1 } & \multicolumn{2}{c}{ Scenario 2 } \\
$j$ & Data rate (bps) & Price & Data rate (bps) & Price \\
\hline$(1)$ & 50 & 100 & 50 & 70 \\
$(2)$ & 70 & 100 & 70 & 75 \\
$(3)$ & 70 & 100 & 20 & 70 \\
$(4)$ & 20 & 100 & 60 & 90 \\
$(5)$ & 60 & 100 & 40 & 60 \\
$(6)$ & 40 & 100 & 50 & 50 \\
$(7)$ & 50 & 100 & 40 & 60 \\
$(8)$ & 40 & 100 & 50 & 75 \\
$(9)$ & 50 & 100 & 60 & 55 \\
$(10)$ & 60 & 100 & 40 & 95 \\
$(11)$ & 40 & 100 & 40 & \\
$(12)$ & 40 & 100 & 40 & \\
\hline
\end{tabular}

TABLE 4: Parameter values for simulations.

\begin{tabular}{lc}
\hline Parameter & Values \\
\hline Number of primary networks & 7 \\
Number of SUs & 12 \\
Number of cycles & 3000 \\
Number of channels in each network & 7 \\
Number of ants & 12 \\
$\alpha, \beta$ & 2 \\
\hline
\end{tabular}

TABLE 5: Algorithms' computational complexity considering convergence time.

\begin{tabular}{lr}
\hline Algorithm & Convergence time (mille-sec) \\
\hline PSO & 0.25 \\
FLACSA & 21 \\
ACSA & 25 \\
GA & 30 \\
\hline
\end{tabular}

In $\mathrm{CRHN}$, increasing number of SUs means that the more the SUs are willing to buy the spectrum, the greater the cost of network is and the greater interference towards PUs is. Figure 9 demonstrates overall objective function in comparison with number of SUs in scenarios 1 and 2, respectively. As is illustrated in Figure 9, the overall objective function, which is the sum of cost and interference, increases with the number of increasing SUs. FLACSA optimizes slightly better than ACSA due its FL updating feature. The experiment was conducted on a PC with Core i5 processor and $6 \mathrm{~GB}$ of memory. The computational complexity of considered algorithms is shown in Table 5. The result shows the average elapsed time after 


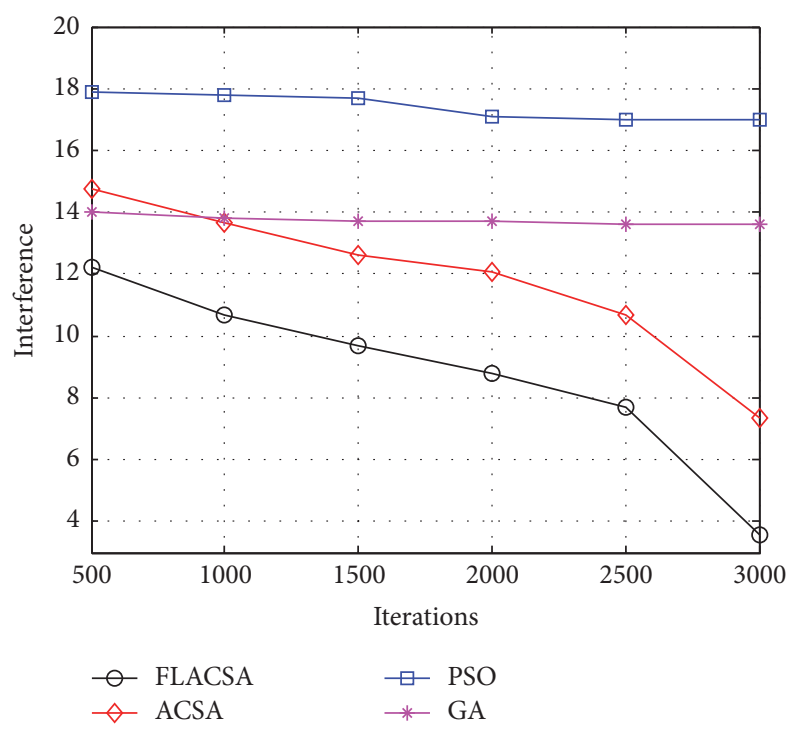

(a)

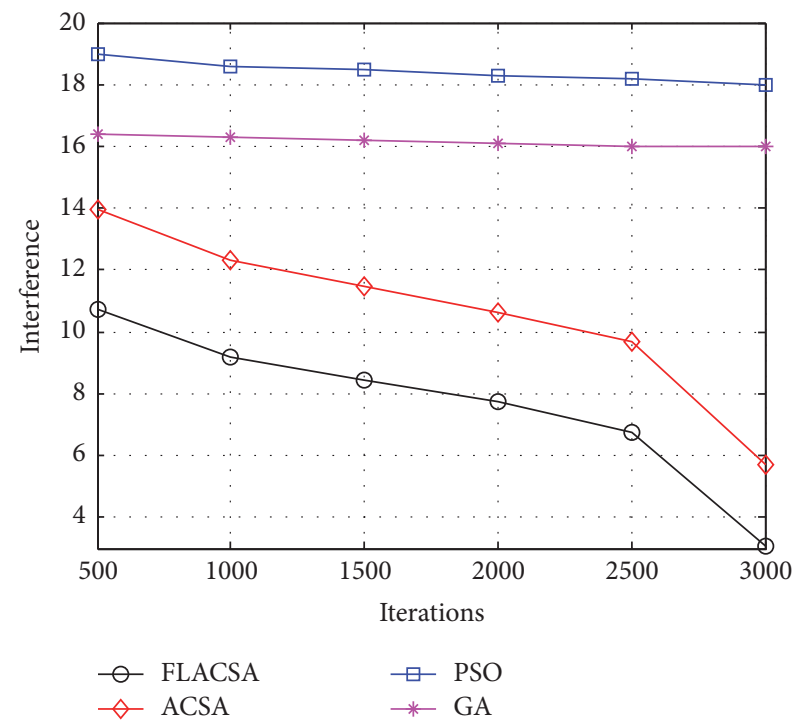

(b)

FIGURE 6: Average accumulative interference comparison in (a) scenario 1 and (b) scenario 2.

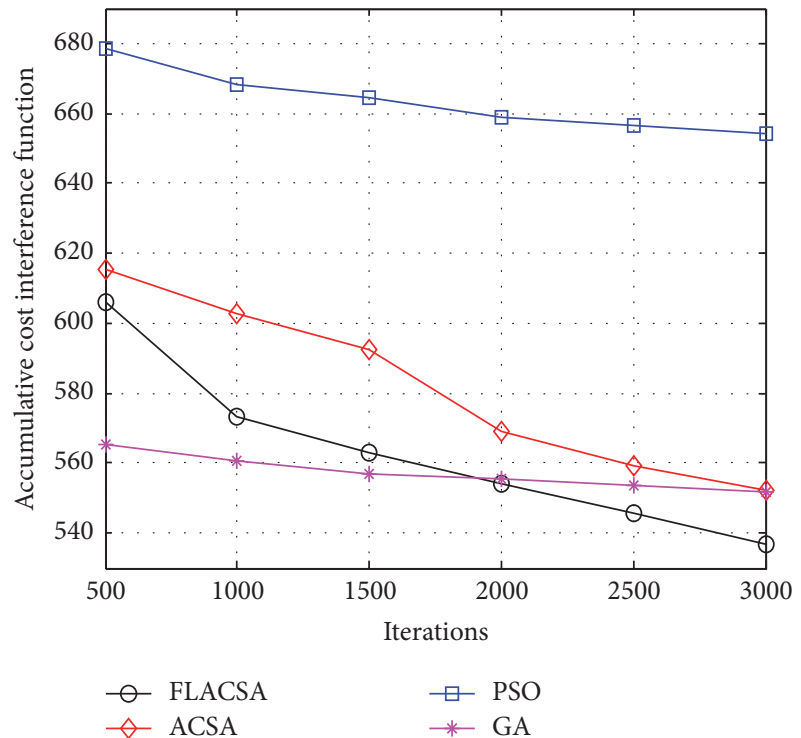

(a)

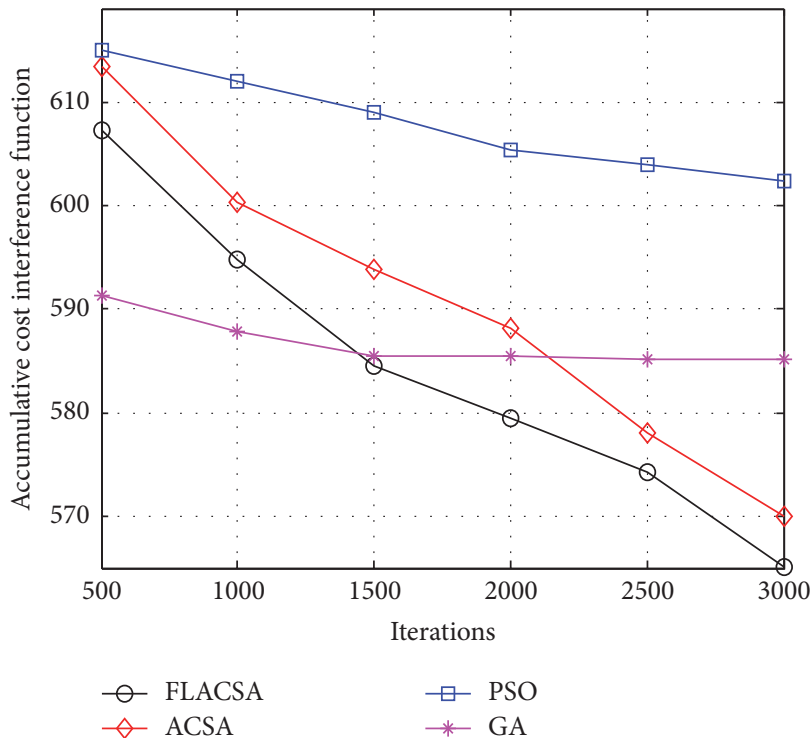

(b)

FIgURE 7: Average objective function in (a) scenario 1 and (b) scenario 2.

100 iterations; PSO converges faster in comparison with other algorithms due to global exploitation capabilities.

GA is slower in comparison with other algorithms due to its more exploration capabilities. The average convergence times of FLACSA and ACSA are $21 \mathrm{msec}$ and $25 \mathrm{msec}$, respectively. Hence, the proposed FLACSA due to low computational complexity as well as low-cost solution can be implemented easily in real-time scenarios.
Figure 10 shows accumulative data rates of SUs against the number of iterations. As shown in Figure 10, the SUs accumulative reward in CRHN increased as the number of iterations increased and converges to the optimal value of 590. FLACSA converges quickly as compared to ACSA due to FL updating feature. Figure 11 shows the impact of varying density of SUs on data rates of SUs in CRHN. As is evident from Figure 11, the SUs individual data rates reduce with the 


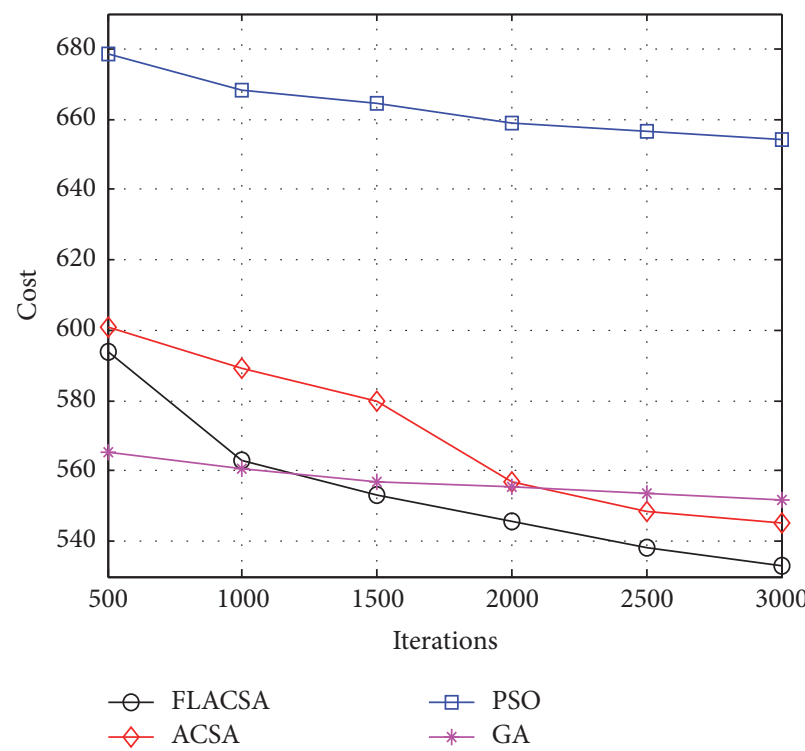

(a)

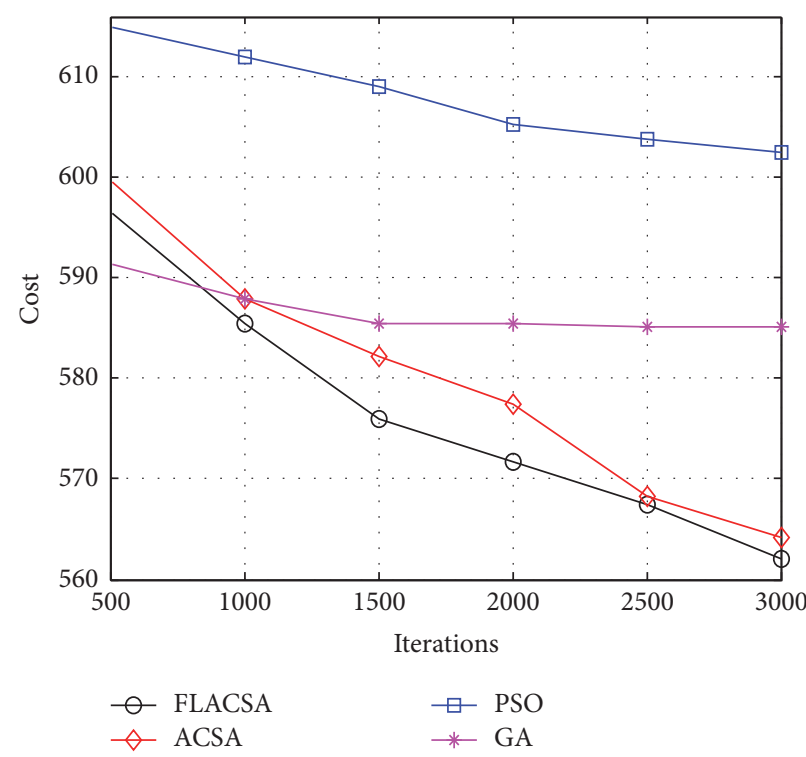

(b)

FIGURE 8: Average accumulative cost against the number of iterations in (a) scenario 1 and (b) scenario 2.

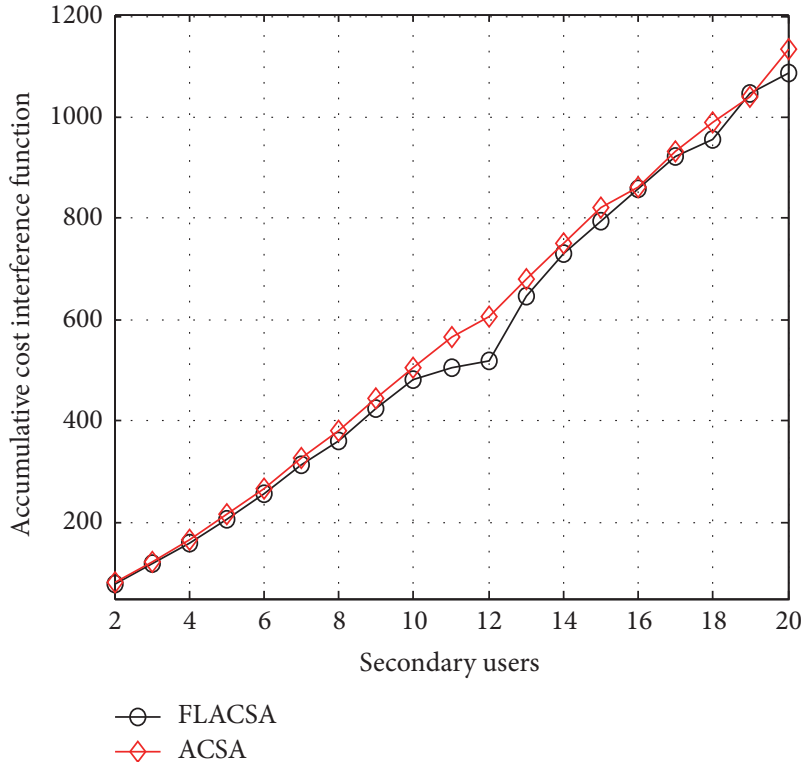

(a)



(b)

FIGURE 9: Average accumulative objective cost and interference function against varying number of SUs in (a) scenario 1 and (b) scenario 2.

increasing number of SUs. The FLACSA performs better than ACSA to optimize data rates of SUs.

\section{Conclusion}

The CRHN is a proposed technology for 5G networks. This research work presents fuzzy ants based optimization method to minimize the interference of SUs incurred to primary networks, reduce the cost to buy primary network resources, and maximize the data rates of SUs. The performance of proposed fuzzy logic based ant colony system algorithm (FLACSA) is evaluated in comparison with other bioinspired algorithms in literature. Moreover, the impact of number of varying SUs in cognitive radio heterogeneous network on data rates is investigated. Furthermore, the SUs accumulative data rate is optimized. The simulation results showed that FLACSA achieves superior results due to fuzzy logic updating the local pheromone in ACSA and can enhance the performance of cognitive radio heterogeneous network further. 


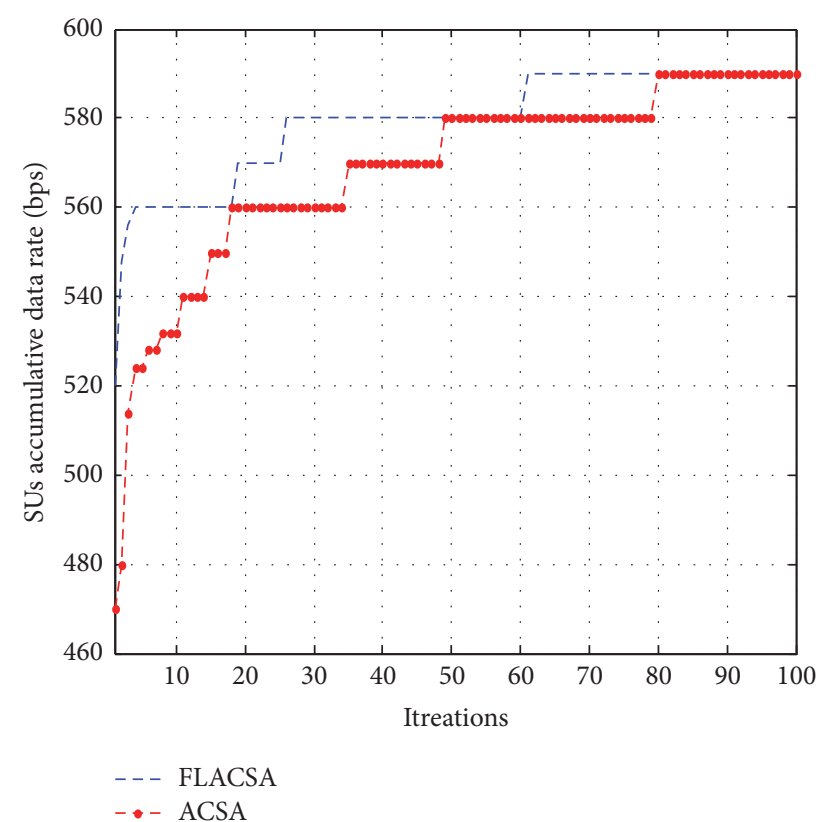

FIGURE 10: SUs accumulative data rate against the number of iterations.

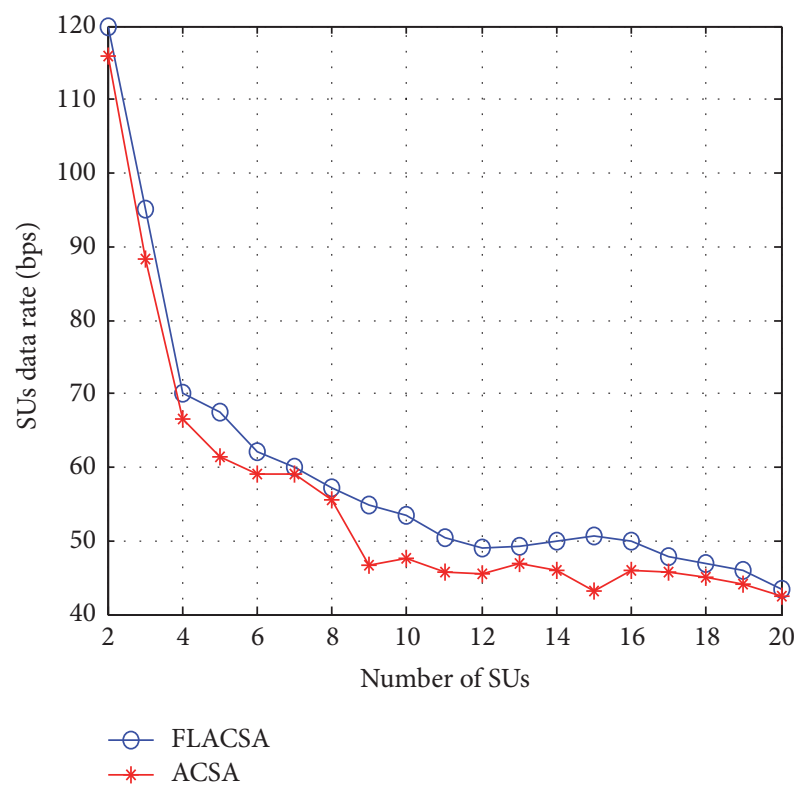

FIGURE 11: SUs data rates against increasing number of SUs in CRHN.

\section{Conflicts of Interest}

The authors declare that they have no conflicts of interest.

\section{References}

[1] J. Mitola III and G. Q. Maguire Jr., "Cognitive radio: making software radios more personal," IEEE Personal Communications, vol. 6, no. 4, pp. 13-18, 1999.
[2] I. F. Akyildiz, W. Lee, M. C. Vuran, and S. Mohanty, "NeXt generation/dynamic spectrum access/cognitive radio wireless networks: a survey," Computer Networks, vol. 50, no. 13, pp. 21272159, 2006.

[3] S. Andreev, M. Gerasimenko, O. Galinina et al., "Intelligent access network selection in converged multi-radio heterogeneous networks," IEEE Wireless Communications, vol. 21, no. 6, article no. A18, pp. 86-96, 2014.

[4] M. Ju and K.-M. Kang, "Cognitive radio networks with secondary network selection," IEEE Transactions on Vehicular Technology, vol. 65, no. 2, pp. 966-972, 2016.

[5] Y. Yang, S. Aïssa, and K. N. Salama, "Spectrum Band Selection in Delay-QoS Constrained Cognitive Radio Networks," IEEE Transactions on Vehicular Technology, vol. 64, no. 7, pp. 29252937, 2015.

[6] L.-C. Tseng, F.-T. Chien, D. Zhang, R. Y. Chang, W.-H. Chung, and C. Y. Huang, "Network selection in cognitive heterogeneous networks using stochastic learning," IEEE Communications Letters, vol. 17, no. 12, pp. 2304-2307, 2013.

[7] T. Leanh, M. Van Nguyen, C. T. Do, C. S. Hong, S. Lee, and J. P. Hong, "Optimal network selection coordination in heterogeneous Cognitive Radio Networks," in Proceedings of the 27th International Conference on Information Networking, ICOIN 2013, pp. 163-168, tha, January 2013.

[8] V. Shrivastav, S. K. Dhurandher, I. Woungang, V. Kumar, and J. J. P. C. Rodrigues, "Game theory-based channel allocation in cognitive radio networks," in Proceedings of the 59th IEEE Global Communications Conference, GLOBECOM 2016, usa, December 2016.

[9] C. Yi, J. Cai, and G. Zhang, "Spectrum Auction for Differential Secondary Wireless Service Provisioning with TimeDependent Valuation Information," IEEE Transactions on Wireless Communications, vol. 16, no. 1, pp. 206-220, 2017.

[10] D. Yang, G. Xue, and X. Zhang, "Group Buying Spectrum Auctions in Cognitive Radio Networks," IEEE Transactions on Vehicular Technology, vol. 66, no. 1, pp. 810-817, 2017.

[11] X. Li, H. Ding, M. Pan, Y. Sun, and Y. Fang, "Users First: ServiceOriented Spectrum Auction With a Two-Tier Framework Support," IEEE Journal on Selected Areas in Communications, vol. 34, no. 11, pp. 2999-3013, 2016.

[12] A. Zhou, B.-Y. Qu, H. Li, S.-Z. Zhao, P. N. Suganthan, and Q. Zhangd, "Multiobjective evolutionary algorithms: a survey of the state of the art," Swarm and Evolutionary Computation, vol. 1, no. 1, pp. 32-49, 2011.

[13] R. A. Rashid, A. H. F. B. A. Hamid, N. Fisal et al., "Efficient inband spectrum sensing using swarm intelligence for cognitive radio network," Canadian Journal of Electrical and Computer Engineering, vol. 38, no. 2, Article ID 7106032, pp. 106-115, 2015.

[14] S. Latif, S. Akraam, and M. A. Saleem, "Channel assignment using differential evolution algorithm in cognitive radio networks," International Journal of Advanced and Applied Sciences, vol. 4, pp. 160-166, 2017.

[15] N. Ul Hasan, W. Ejaz, N. Ejaz, H. S. Kim, A. Anpalagan, and M. Jo, "Network Selection and Channel Allocation for Spectrum Sharing in 5G Heterogeneous Networks," IEEE Access, vol. 4, pp. 980-992, 2016.

[16] M. Dorigo, V. Maniezzo, and A. Colorni, "Ant system: optimization by a colony of cooperating agents," IEEE Transactions on Systems, Man, and Cybernetics B: Cybernetics, vol. 26, no. 1, pp. 29-41, 1996.

[17] L. A. Zadeh, "Fuzzy sets," Information and Control, vol. 8, no. 3, pp. 338-353, 1965. 
[18] P. Chemouil, "A Fuzzy Control Approach for Adaptive Traffic Routing: A new approach to traffic routing is essentially based on heuristic rules derived from expert knowledge and human experience. This approach has been used to develop a fuzzy routing system applied to a model of the French long-distance telephone network," IEEE Communications Magazine, vol. 33, no. 7, pp. 70-76, 1995.

[19] E. H. Mamdani, "Application of fuzzy algorithms for the control of a simple dynamic plant," Proceedings of the IEEE, vol. 121, no. 12, pp. 1585-1588, 1974.

[20] M. Dorigo and L. M. Gambardella, "Ant colony system: a cooperative learning approach to the traveling salesman problem," IEEE Transactions on Evolutionary Computation, vol. 1, no. 1, pp. 53-66, 1997. 




\section{Enfincering}
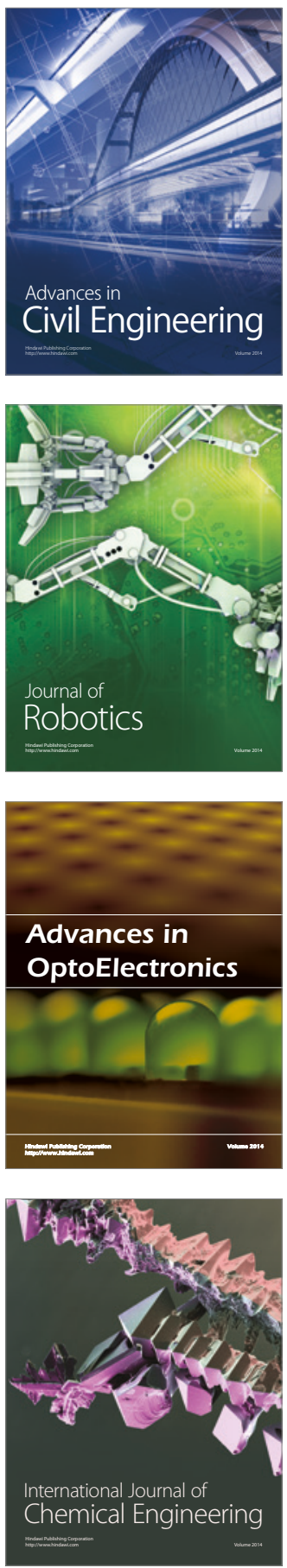



The Scientific World Journal

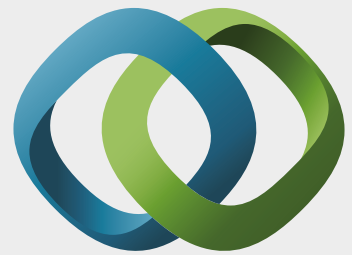

\section{Hindawi}

Submit your manuscripts at

https://www.hindawi.com
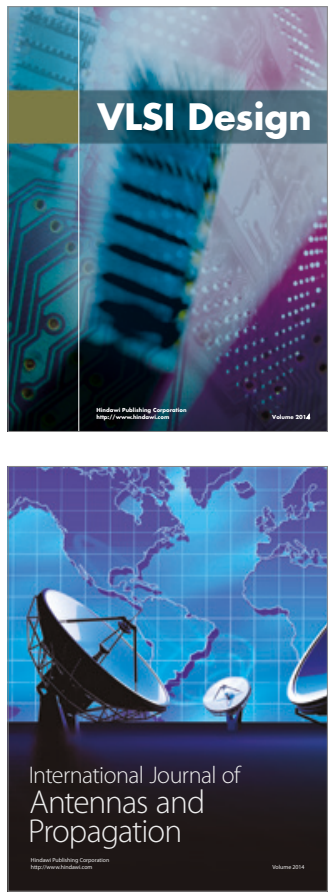

\section{Rotating}

Machinery


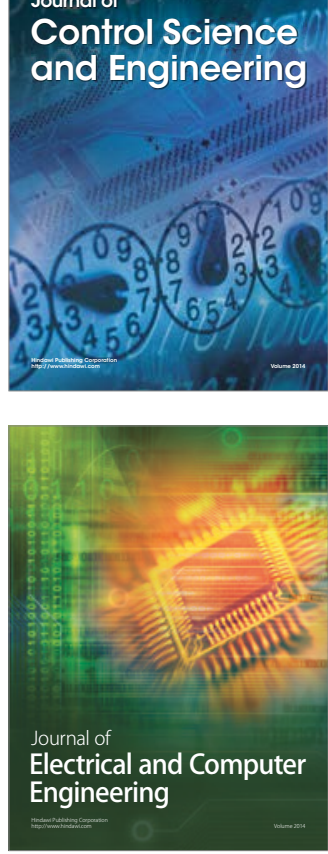

Distributed

Journal of

Control Science

and Engineering
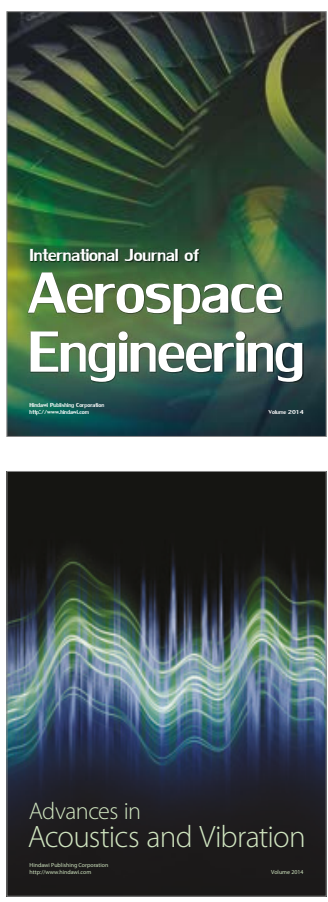

Sensor Networks 